\title{
The Sedimentation Rate of Free Erythrocytes and Their Associates, the Influence of a Standing Ultrasonic Wave
}

\author{
Valeri A. Doubrovski ${ }^{1}$, Sergey V. Markov ${ }^{1,2^{*}}$, Stanislav O. Torbin ${ }^{1}$, and Elena P. Karpocheva ${ }^{3}$ \\ ${ }^{1}$ Department of Medical and Biological Physics, Saratov Medical State University, 112 Bolshaya Kazachya str., Saratov, \\ 410012, Russia \\ ${ }^{2}$ Saratov State University, 83 Astrakhanskaya str., Saratov 410012, Russia \\ ${ }^{3}$ Saratov Regional Blood Transfusion Center, 27 Gvardeyskaya str., Saratov 410033, Russia \\ * e-mail: markovsergeyv@inbox.ru
}

\begin{abstract}
A comparative analysis of the sedimentation rates of free erythrocytes and their associates - aggregates and agglutinates was carried out. The influence of a standing ultrasonic (US) wave on the blood sedimentation process was investigated. The problem was dictated not only by a purely scientific interest in the sedimentation of micro-objects in a liquid medium, but from a practical point of view also - the development of an instrumental acousto-optical method for human blood group typing. Registration of the blood sedimentation process was carried out by means of digital video recording with subsequent computer processing of the photographic images obtained. The magnitude of blood sedimentation rate was experimentally determined for various samples and conditions: 1) blood solutions with / without ultrasound exposure for different time dosages; 2) a mixture of blood samples with hemagglutinating substances (Tsoliklon) without / with ultrasonic action both for a positive agglutination reaction (formation of agglutinates) and negative ones (absence of agglutinates). Two approaches were used to process the photographic images: 1) in the case of agglutinates sedimentation the discrete counting and analysis of agglutinate parameters were used; 2) in other cases, the blood sedimentation rate was determined by the movement of the "supernatant-sediment" (plasma solution - erythrocytes mass) boundary. For the experimental conditions accepted the blood sedimentation rates were compared and, correspondently, the influence of the ultrasonic standing wave upon the process under study was estimated. It was shown that in the presence of ultrasound the sedimentation rate of $\mathrm{RBC}$ agglutinates (positive agglutination reaction) is more than an order higher than the sedimentation rate of free erythrocytes and their aggregates (negative agglutination reaction). Such a great difference in the sedimentation rates of agglutinates in comparison with free erythrocytes and their aggregates makes it possible to obtain a high degree of resolution of acousto-optical method for instrumental blood typing, and, hence, high reliability in blood group determination. (C) 2021 Journal of Biomedical Photonics \& Engineering.
\end{abstract}

Keywords: blood typing; sedimentation; blood; ultrasound; sonication; agglutination; optical registration.

Paper \#3410 received 15 Mar 2021; revised manuscript received 9 Jul 2021; accepted for publication $11 \mathrm{Jul}$ 2021; published online 28 Jul 2021. doi: 10.18287/JBPE21.07.040501. 


\section{Introduction}

Ultrasonic waves are widely used in clinical medicine [1], for example, ultrasound therapy and surgery, Doppler spectroscopy, ultrasound investigations. In parallel, the investigations are underway to use ultrasound in the field of biomedical technologies and laboratory diagnostics, for example, to control particles [2-4] and cells [4-7] in suspensions, to filter bacterial cells [8-11] and their separation [12]. A promising direction is to use ultrasound for the aims of biomedical immunological researches [13-20]. Note that immunological methods based on specific antigens antibodies interactions in vitro with subsequent observation of the immune complexes formed are the basis of many medical laboratory diagnostic tests, in particular, for the instrumental human blood group determination.

It was for the first time when the authors were involved into the problem of automatic device creation for blood typing based on the use of a probing optical beam and stimulating ultrasonic radiation simultaneously in 1986 [13]. In the further studies and developments this method was carried out in analog [18-20] or digital forms [21-29] and in the whole it was named as "acousto-optical method for blood group determination".

An ultrasonic standing wave, oriented in the vertical direction, is grouping erythrocytes (RBC) in its nodal zones, what leads to the cells stratification in solution with a period equal to a half of the ultrasonic wavelength. The convergence of erythrocytes in the standing wave nodes increases the probability of their induced aggregation. In the presence of immunologically adequate hemagglutinating serum (or monoclonal antibodies), erythrocytes form large immune complexes - agglutinates ("positive reaction" of agglutination). These complexes, like "free" erythrocytes or their aggregates are levitating - they are held in suspension by an ultrasonic wave. The ultrasound turning off leads to a rapid sedimentation of large and rather strong $\mathrm{RBC}$ agglutinates. As a result, the biological object under investigation becomes transparent quickly and significantly.

In the case of immunological inconformity of the blood sample under study with the type of hemagglutinating serum or monoclonal antibodies ("negative reaction"), the formation of agglutinates in the nodes of ultrasonic standing wave is not observed, weak RBC aggregates are formed only, which basically disintegrate into individual cells when the ultrasound is turned off. Since the sedimentation rate of free erythrocytes is much lower than the rate of agglutinates, then free erythrocytes remain suspended for a long time in respect to agglutinates, and that is why the biological medium remains turbid, strongly absorbing and scattering the probing light beam. Naturally, the difference in optical properties of the samples under study (for example, optical density or transmittance) indicates the nature of the interaction of cells and the type of reaction, which ultimately makes it possible to determine the blood group of the sample. Note that the greater the difference in optical parameters for positive and negative agglutination reactions - the higher the "resolution" of the method, and, consequently, the higher the reliability of blood group sample determination.

Thus, the acousto-optical method of blood typing is based on two physical phenomena: the grouping of erythrocytes by a standing ultrasonic wave and the sedimentation of erythrocytes or their associates (RBC aggregates or agglutinates) after ultrasound turning off. The visualization of RBC grouping process and its modeling were carried out in Ref. [29] and Refs. [18 - 20,29], respectively. The aim of this work is to visualize and study the features of the second component of the acousto-optical method of blood typing:

- measurement of the sedimentation rate of erythrocytes and their associates after ultrasound irradiation in comparison with the similar studies, but without acoustic action;

- quantitative comparison of sedimentation rates of different blood samples under different experimental conditions;

- the quantitative estimation of the magnitude of the acousto-optical method resolution of blood typing and its relationship with the sedimentation rate of biological object under study.

\section{Objects and experimental technique}

The research objects are:

- blood solutions in saline of various dilution levels (with / without ultrasound);

- blood solutions containing erythrocytes and naturally formed RBC aggregates, as well erythrocyte aggregates formed by ultrasound standing wave at a negative agglutination reaction;

- RBC agglutinates formed by/without ultrasonic wave action.

Experiments to record the process of blood solution sedimentation were carried out on the setup shown in Fig. 1a.

The dimensions of the internal cavities of the rectangular cuvettes used are $10 \times 10 \times 45 \mathrm{~mm}$ (experiments without monoclonal antibodies) or $5 \times 18 \times 32 \mathrm{~mm}$ (experiments with the participation of monoclonal antibodies). In the second case, a narrower ( $5 \mathrm{~mm}$ thick) cuvette was used to visualize individual large erythrocyte agglutinates and their subsequent computer processing. The biological object was probed by collimated radiation of an LXHL-G1S type LED, the spectrum of which corresponded to the absorption spectrum of hemoglobin in the green region (Fig. 1b). The selection of the probing light radiation spectrum was dictated by the need to increase the contrast of the photographic image boundary "plasma solution-suspension of erythrocytes", which contributes to an increase of the blood sedimentation rate accuracy measured. The probing light beam felt down 
upon the Logitech QuickCam polychrome webcam after passing through the sample studied. The resultant videos were cropped into separate frames (files), which were decomposed then into RGB components and Green $(\mathrm{G})$ component only was analyzed. The choice of the $\mathrm{G}$ component of the photographic image was associated with the absorption spectrum of light by hemoglobin in the green region and. naturally, with the spectrum of the probing radiation of the LED (Fig. 1b).

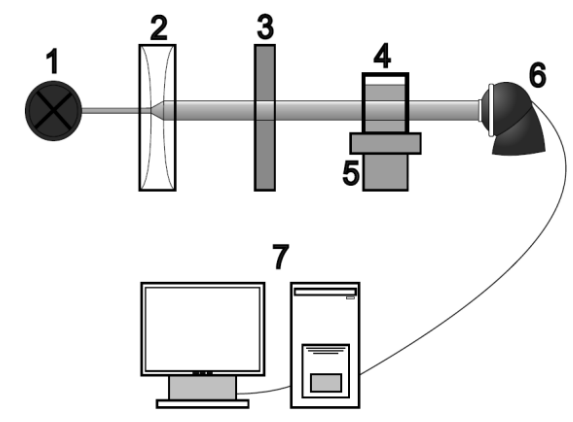

(a)

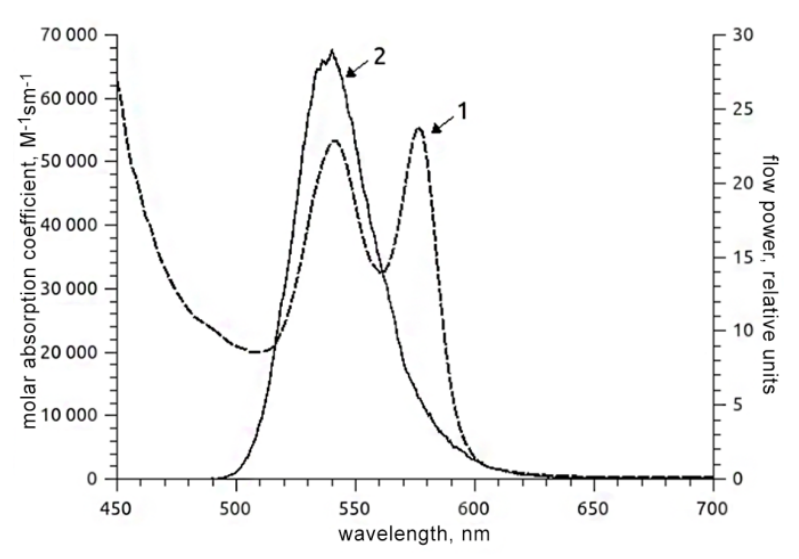

(b)

Fig. 1 The experimental setup (a) and the probing radiation and the biological object's spectra (b): a) 1 - light source (LED); 2 - telescopic lens system; 3 - light filter; 4 - cuvette with the test solution; 5 - table for fixing cuvettes; 6-digital video camera; 7 - computer; b) 1 - absorption spectrum of hemoglobin (left ordinate), 2-LED emission spectrum (right ordinate).

The experimental technique, the registration of blood sedimentation process and the computer processing highly depended on the nature of the object and the properties of its photographic image.

\section{Sedimentation of erythrocytes and their aggregates under ultrasound action}

The study of the objects sedimentation features and determination of the sedimentation rate for erythrocytes and /or their associates, it was revealed:
- there is a fairly clear boundary between the supernatant (supernatant fluid-plasma solution) and sediment (sediment-erythrocytes and erythrocyte aggregates) (Fig. 2);

- let us get ahead-such a boundary is fundamentally absent at a positive agglutination reaction stimulated by ultrasound; naturally, it makes difficult to estimate the rate of blood sedimentation for such a case (to be discussed in Section 4.4).

\section{US time duration $\mathrm{t}=60 \mathrm{~s}$}

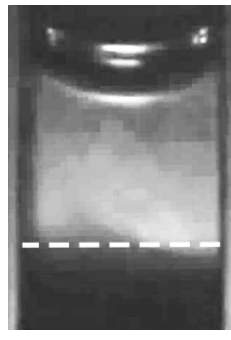

(a)

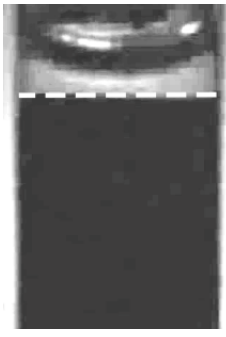

(b)

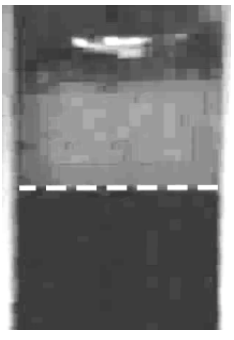

(c)

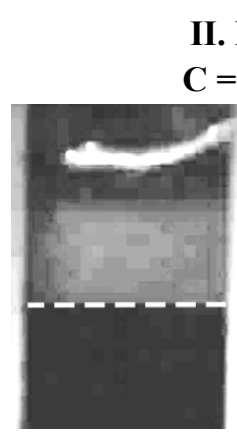

(d)
II. Blood concentration

$\mathrm{C}=\mathbf{1 0 0 \%}$ (whole blood)

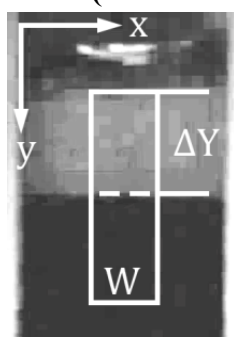

(e)

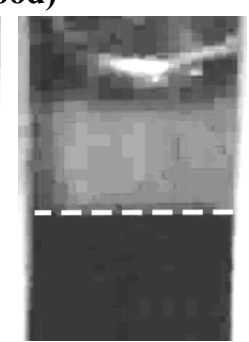

(f)
Fig. 2 The images of samples after the standing ultrasound wave action for different content of the whole blood in solution: a) $1 \%$, b) $30 \%$, c) $100 \%$, or different time duration of ultrasound action d) 30 , e) 60, f) $90 \mathrm{sec}$. The experimental results illustrated for the case when monoclonal antibodies were not applied. The series of images $(\mathrm{a}-\mathrm{c})$ correspond to the time duration of ultrasonic action upon the blood samples $\mathrm{t}=60 \mathrm{sec}$, and the series $(\mathrm{d}-\mathrm{f})$ was obtained for the whole blood. The images $(\mathrm{a}-\mathrm{c})$ correspond to the moment $60 \mathrm{sec}$ after the ultrasound is turned off. Dotted lines are additionally drawn to improve the visualization of the supernatant - sediment boundary. The $\Delta \mathrm{Y}$ value is the displacement of the "supernatant - sediment" boundary during blood sedimentation, $\mathrm{W}$ is the area of computer processing of photographic images.

Photos (Fig. 2) indicate a rather strong dependence of the sedimentation rate on the concentration of erythrocytes in the blood solution in the presence of ultrasound. The processing of photographic images presented in Fig. 2 gives the dependence of blood sedimentation rate $\mathrm{v}=\Delta \mathrm{Y} / \Delta \mathrm{t}$ on the degree of the whole blood dilution in saline (the proportion of blood in saline in \%) (Fig. 3). 


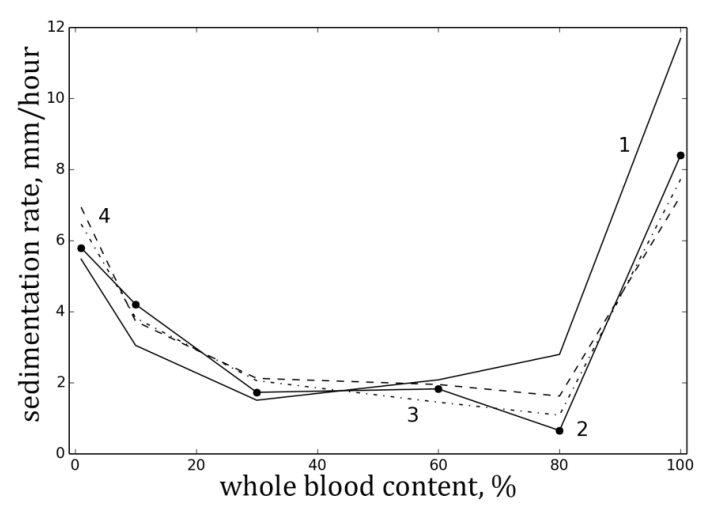

Fig. 3 The dependence of the sedimentation rate of blood solutions on whole blood content at various ultrasonic action duration: 1) $\mathrm{t}=0 \mathrm{sec}$ (no ultrasonic action), 2) $t=30 \mathrm{sec}, \quad 3) \mathrm{t}=60 \mathrm{sec}, \quad 4) \mathrm{t}=90 \mathrm{sec}$. Hemagglutinating antibodies (Tsoliklon) are absent; Graph 1 is borrowed from Ref. [30].

It is interesting to note that blood solution sedimentation rate without ultrasound action is minimal, when the whole blood content in the sample is in the range of $30-50 \%$. At strong blood dilutions up to $0.25 \%$ of whole blood, the rate of its sedimentation increases significantly and is approximately equal to the sedimentation rate of an individual RBC cell. This is due to the fact that for such significant blood dilutions, the erythrocytes are so far apart from each other that the distance between them significantly exceeds the size of erythrocytes themselves. In this case, the velocity of the upcoming plasma solution flow displaced by erythrocytes sedimenting is not high; therefore, the inhibitory effect of this flow upon erythrocytes is small and, as a result, the RBC sedimentation rate increases [30]. For the same reason (the remoteness of erythrocytes from each other), the influence of ultrasound standing wave on the blood solution sedimentation rate is not too noticeable.

For weak blood dilutions by saline (quasi the whole blood) in the absence of ultrasound the blood solution sedimentation rate increases for the same reason (Fig. 3, Graph 1), but this time it takes place due to the increase of the distance between the erythrocytes aggregates formed spontaneously. Indeed, an increase in the proportion of the whole blood in saline over $80 \%$ increases the probability of large aggregates forming. In this case, the distance between the aggregates increases, the rate of the upcoming plasma solution flow decreases, and the rate of blood sedimentation, respectively, increases [30].

In this range of erythrocyte concentrations, the effect of ultrasound is more noticeable, since the standing wave is stimulating to enlarge the RBC aggregates and, accordingly, the distance between them increases. As a result, the sedimentation rate of $\mathrm{RBC}$ aggregates increases in comparison to the samples at a whole blood content about $30-80 \%$. At the same time, in comparison with the case of blood sedimentation without ultrasound action in the blood content range $80-100 \%$ ultrasound does not increase the sedimentation rate, but, in contrary, decreases it. Multiple visual observations of blood solution sedimentation process after ultrasound action show that, when ultrasound is turned off, the aggregates formed are induced to sediment rather sharply at the initial moment, creating a certain upcoming fluid flow but at the same time they become rapidly destroyed - erythrocytes become free; that is why they sediment in about the same way as without ultrasound action. Moreover, the formed upcoming flow entrains a certain amount of erythrocytes, creating a certain obstacle to the process of blood sedimentation, which reduces the rate of its sedimentation.

\section{Erythrocyte agglutinates sedimentation}

\subsection{Experimental technique, visualization of erythrocyte grouping by a standing ultrasonic wave}

In experiments on the registration of blood sedimentation after positive or negative reactions of RBC agglutination with or without ultrasonic standing wave action, donor blood solutions of types $\mathrm{A} \beta$ and $\mathrm{B} \alpha$, as well as the solutions of anti-A and anti-B monoclonal antibodies (ERYTHROTEST ${ }^{\mathrm{TM}}$-Tsoliklones) were used. Recall that the agglutination reaction is considered as positive if the result is an immune RBC complex - agglutinate ("+" in Table 1), otherwise the reaction is considered as negative ("-") in Table 1).

Table 1 - Human blood typing using tsoliclones.

\begin{tabular}{|c|c|c|}
\hline \multicolumn{2}{|c|}{ Tsoliclones } & \multirow{2}{*}{$\begin{array}{c}\text { The tested } \\
\text { blood belongs } \\
\text { to the group }\end{array}$} \\
\cline { 1 - 2 } anti-A & anti-B & \\
\hline $\begin{array}{c}\text { The RBC reaction type under } \\
\text { investigation }\end{array}$ & $0(\mathrm{I})$ \\
\hline- & - & $\mathrm{A}(\mathrm{II})$ \\
\hline+ & - & $\mathrm{B}(\mathrm{III})$ \\
\hline- & + & $\mathrm{AB}(\mathrm{IV})$ \\
\hline+ & + & \\
\hline
\end{tabular}

The components of the mixture "blood solution - hemagglutinating substance" were mixed in a cuvette mounted on a piezoceramic transducer. The dimensions of the rectangular cuvette were $5 \times 18 \times 32 \mathrm{~mm}$, and the probing optical beam (Fig. 1a) fell on the cell wall with a maximum area of $18 \times 32 \mathrm{~mm}$. In the absence of ultrasound, a digital video camera was turned on in $10 \mathrm{~s}$ after mixing the components and the observed process was recorded.

In the presence of ultrasound, the erythrocytes grouping in the nodes of the standing wave occurred (it was noted in Section 1 Introduction) and, as a result, stratification of blood sample both for a positive or a negative agglutination reaction had taken place. The process of stratification of a suspension of erythrocytes, 
and then the destruction of these layers due to erythrocyte aggregates sedimentation (negative agglutination reaction) is shown in Fig. 4. The initial moment of time, when the ultrasound is already on, but the process of stratification of the suspension "blood solution-monoclonal antibodies" has not practically started yet, corresponds to Fig. 4a. That moment of time the erythrocytes are distributed in the cuvette relatively uniformly, the cell suspension absorbs the light flux emitted by the LED with $\lambda \pm \Delta \lambda=540 \pm 20 \mathrm{~nm}$ (Fig. 1b) efficiently, so the brightness of the photo image is close to zero.

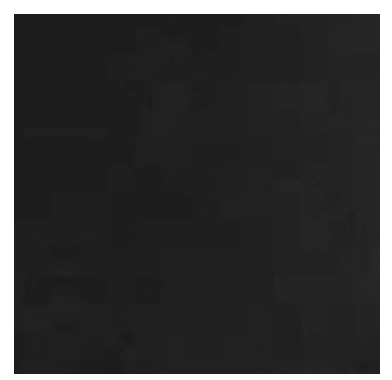

(a)

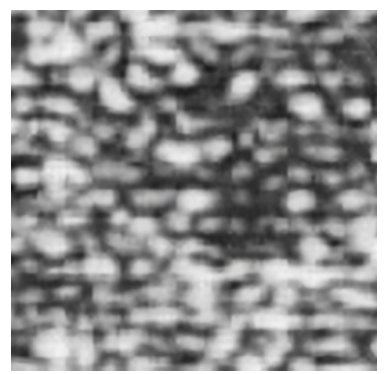

(c)

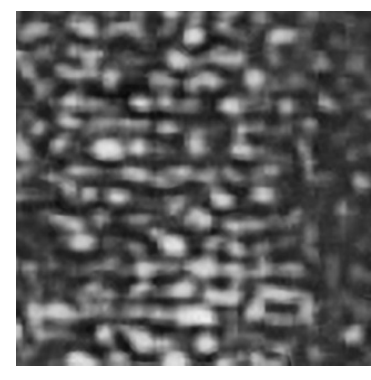

(b)

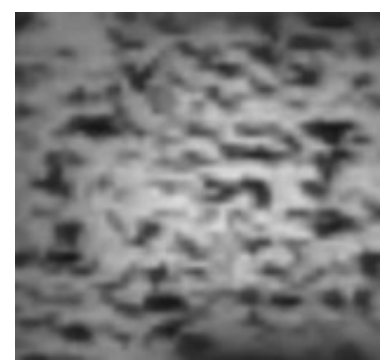

(d)
Fig. 4 The dynamics of erythrocytes grouping by a standing ultrasonic wave for the case of a negative agglutination reaction. The photo images in $\mathrm{G}$ channel of "blood solution-monoclonal antibodies" suspension during its irradiation by a standing ultrasound wave at different moments of time in respect to the start of ultrasound action are presented: $a) t=0 \mathrm{sec}, \mathrm{b}) \mathrm{t}=15 \mathrm{sec}$, c) $\mathrm{t}=50 \mathrm{sec}, \mathrm{d}) \mathrm{t}=226 \mathrm{sec}$.

Fig. $4 \mathrm{~b}$ demonstrates the stratification of the cell suspension at the $50^{\text {th }} \mathrm{sec}$ after the ultrasound is switched on: the dark stripes correspond to the areas of grouped erythrocytes that intensively absorb light; light stripes - areas with a small number of cells, light passes relatively freely through these areas. The layers of erythrocytes levitate, being held by a standing ultrasound wave, and some disturbance in the periodicity of the bands is caused by not an ideal distribution of the ultrasound field in the cuvette. By the $50^{\text {th }} \mathrm{sec}$ (Fig. 4c), the erythrocytes, being grouped in the nodes of the standing ultrasound wave, have formed such large aggregates that the ultrasound field is unable to hold such heavy aggregates, their layers are mixing, some of the aggregates are sedimenting, the image becomes less regular. The final destruction of the erythrocytes stratification picture can be seen in Fig. $4 \mathrm{~g}\left(226^{\text {th }} \mathrm{sec}\right)-$ a few seconds before the ultrasound was turned off.

\subsection{Experimental results}

A more complete gallery of photographs - not only for a negative agglutination reaction (Fig. 2), but for a positive one also, as well as with and without the participation of an ultrasound standing wave is presented in Fig. 5.

\section{Agglutination reaction}

positive

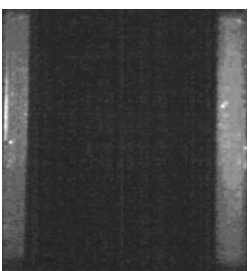

(a)

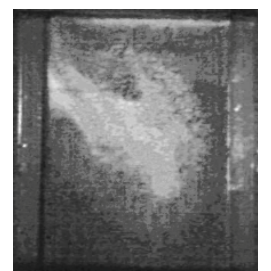

(b)

negative

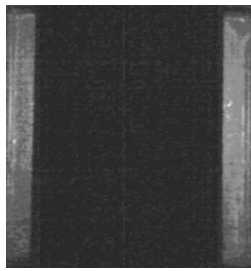

(d)

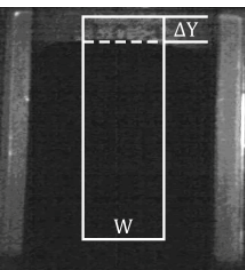

(e)

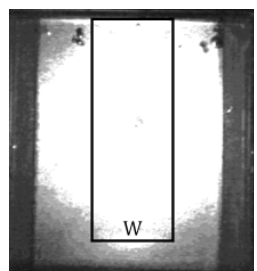

(c)

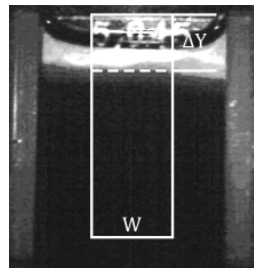

(f)
Fig. 5 Photographs of blood samples for a positive and negative agglutination reactions without ultrasonic action on a biological object and with the participation of ultrasound. Photo (a) and (d) - initial photographic images (for comparison with subsequent ones). Photographs for positive (b) and negative (e) agglutination reactions without ultrasound action on the mixture by the $14^{\text {th }}$ and $54^{\text {th }}$ min respectively. Photographs for positive (c) and negative (e) agglutination reactions at ultrasonic irradiation of the mixture by the $4^{\text {th }}$ and $31^{\text {st }}$ min. respectively. The whole blood content in the experiments was $\sim 2 \%$. The time duration of ultrasonic wave action upon the mixture was 50 sec. W - area for computer processing; $\Delta \mathrm{Y}$ - displacement of the "supernatant - sediment" border.

Fig. 5 shows the following.

\section{A) For a negative agglutination reaction:}

- Regardless of the suspensions irradiation by ultrasound or for its absence, the "supernatant-sediment" boundary is observed; the temporary movement of it gives information on the sedimentation rate of the blood sample solution. 
- The grouping of erythrocytes by a standing ultrasound wave (Fig. 5f) leads to the formation of large aggregates, which increases the rate of blood sedimentation (comparison of Figs. 5e and 5f). Moreover, as the observations during ultrasonic irradiation of "blood solution - monoclonal antibodies" suspension show the blood sedimentation begins to occur even before the ultrasound is turned off, that is, in the process erythrocytes and their aggregates grouping. This is due to the fact that ultrasound forms such large erythrocyte aggregates that a standing ultrasound wave is not able to keep them in a state of levitation.

- In the absence of ultrasound, blood sedimentation does not occur immediately, but after several hundred seconds (delay time $t \approx 700 \mathrm{sec}$, Fig. 5e). This phenomenon was observed consistently with various samples of blood solution. The explanation is based on the experimental observations: the formation of the supernatant - sediment boundary and its subsidence begins only after a portion of plasma solution in the form of a coming up flow in respect to the direction of erythrocytes (their aggregates) movement enters upward along the glass walls of the cuvette and is located above the layer of erythrocyte mass.

\section{B) For a positive agglutination reaction}

The processing of the photographic image corresponding to Fig. 5c is difficult: erythrocyte agglutinates are formed so quickly, their sizes and sedimentation rate are so high that there is no "supernatant - sediment" boundary and, therefore, the principle of processing of photographic images should be different in relation to the case, when such a boundary exists. The principle of the processing of photographic images like Fig. $5 \mathrm{c}$ to find the blood sedimentation rate is described in Section 4.4.

\subsection{The principle of photographic images processing in the presence of a supernatant - sediment interface}

For digital processing of photographic images in Fig. $5 \mathrm{~b}$, e, f, the zones $\mathrm{W}$ with dimensions $5 \times 20 \mathrm{~mm}$ were chosen. For the photographs indicated, in contrast to Fig. 5c, it is fundamental that for these cases there is a "supernatant - sediment" boundary ("plasma solution - erythrocyte mass"). Within the selected zone, the photographic image (video frame) was transformed into a two-dimensional array, the elements of which were the brightness B (brightness) of pixels for the $G$ component of RGB decomposition of the photographic image obtained. To get a more clear determination of the boundary the binarization of the photographic image was carried out: in the array studied the pixel brightness were averaged line by line (from left to the right and from top to bottom). As a result, the two-dimensional array was transformed into a one-dimensional $\mathrm{B}_{\mathrm{av}}(\mathrm{Y})$, which was then filtered according to a given threshold value $\mathrm{B}_{\mathrm{thr}}$, determined from the analysis of the photo image. The elements of a one-dimensional array with the brightness lower or equal to the threshold $\mathrm{B}_{\mathrm{av}}(\mathrm{Y}) \leq \mathrm{B}_{\mathrm{thr}}$ were assigned as the brightness equal to zero $\mathrm{B}_{\mathrm{av}}(\mathrm{Y})=0$, the rest of the elements were assigned as a certain brightness $\mathrm{B}_{\mathrm{av}}=\mathrm{B}_{0}$, different from zero.

In the limits of the zone $\mathrm{W}$ analyzed, each line of the photographic image scanned has its own Y coordinate. Therefore, when reading the results of the onedimensional array, the transition from the "light" to "dark" part of the zone $\mathrm{W}$ indicates the location of the supernatant-sediment boundary. Naturally, such a boundary corresponds to a line of a photographic image with a maximum brightness gradient $\Delta \mathrm{B}_{\mathrm{av}} / \Delta \mathrm{Y}$. The movement of boundary from frame to frame determines the rate of blood sedimentation in (pixels/s). It is possible to find the sedimentation rate of the object $\mathrm{v}=\Delta \mathrm{Y} / \Delta \mathrm{t}$ in $(\mathrm{mm} / \mathrm{min})$, if to use the resolution $\mathrm{r}(\mathrm{r}=38 \mathrm{ps} / \mathrm{mm})$ magnitude of the experimental setup (Fig. 1a). Indeed, the computer processing of the temporary photographic images series Fig. 5 (b, e, f) made it possible to determine the level of the "supernatant-sediment" boundary displacement along $\mathrm{Y}$ axis in time $\mathrm{t}$ (Fig. 6).

Comparison of Fig. 6 shows that the blood sedimentation rate is minimal for a negative agglutination reaction and, at the same time, in the absence of ultrasonic action upon the blood solution (Fig. 6e): there are no factors that would lead to the formation of large erythrocyte associates and in a large number.

Fig. $6 \mathrm{f}$ shows that for negative agglutination reaction the blood sedimentation rate is maximum when during ultrasonic irradiation of an object the ultrasound use to form large erythrocyte aggregates (not agglutinates), which are quickly sedimenting quickly. After that when ultrasound is switched off the fragile aggregates use to disintegrate into individual erythrocytes or smaller aggregates. They are sedimenting just as slowly as free erythrocytes or cells after a negative agglutination reaction (Fig. 6e). This is also evidenced by the fact that the slope of the curves (sedimentation rate) in Figs. 6e and $6 f$ are practically the same.

As it was noted in Section 4.2 for a positive agglutination reaction with the participation of ultrasound (Fig. 6b), the process of RBC agglutinates sedimentation occurs so quickly and rather chaotically that the "supernatant-sediment" boundary is absent. That is why in Fig. $6 \mathrm{c}$ the ordinate shows the value of the "equivalent displacement" of a certain effective boundary "supernatant - sediment" (the introducing and the description of this parameter is presented in Section 4.4). For a positive agglutination reaction but without ultrasound action, the blood sedimentation (Fig. 6b), as in the case of a negative reaction (Fig. 6e), occurs with a time delay (the lag time $\mathrm{t} \approx 350 \mathrm{sec}$; Fig. 6b). Possible explanation: at whole blood content in the sample $\sim 2 \%$ the mean statistical distance between erythrocytes is so great (2-3 times more than the erythrocyte diameter itself) that the probability of an antigen-antibody type of immune interaction (erythrocyte - monoclonal antibodies) with the formation of an erythrocyte complex is small. 


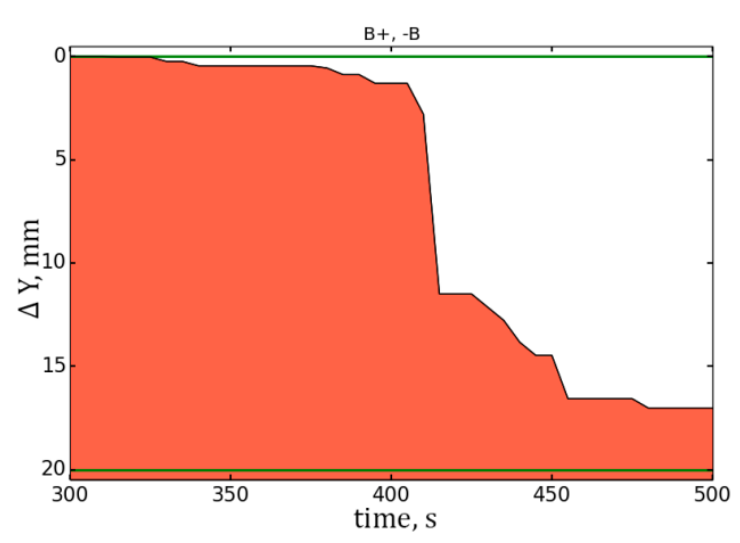

$\mathrm{b}$

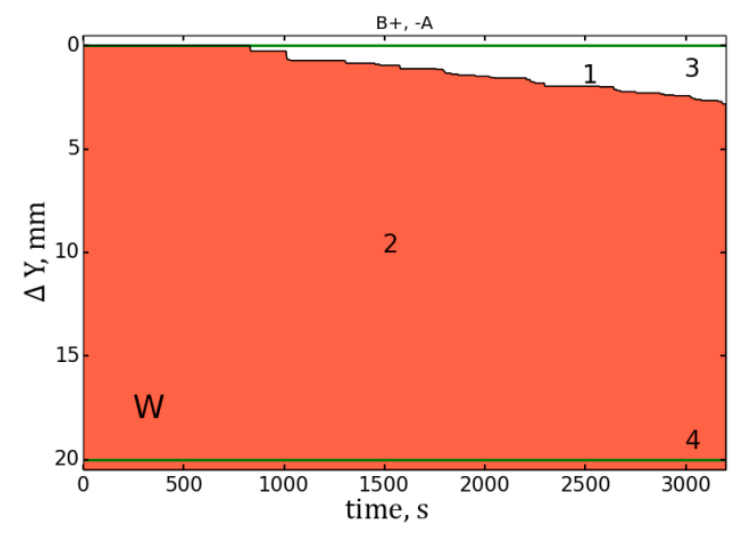

e

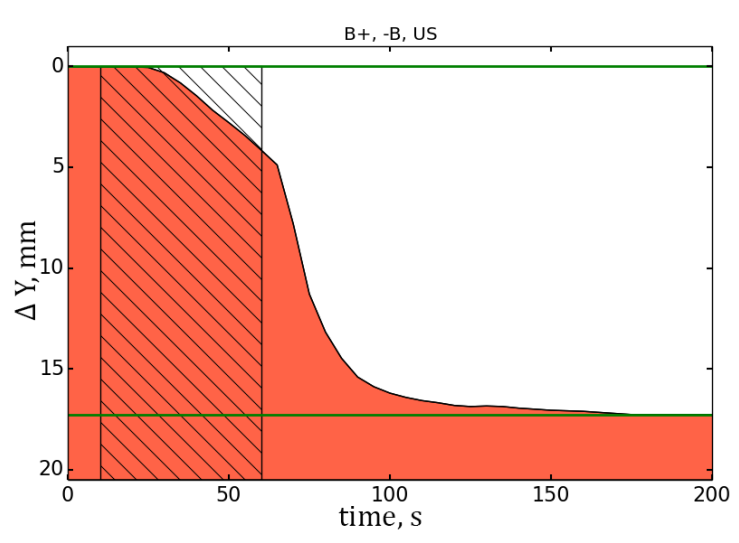

C

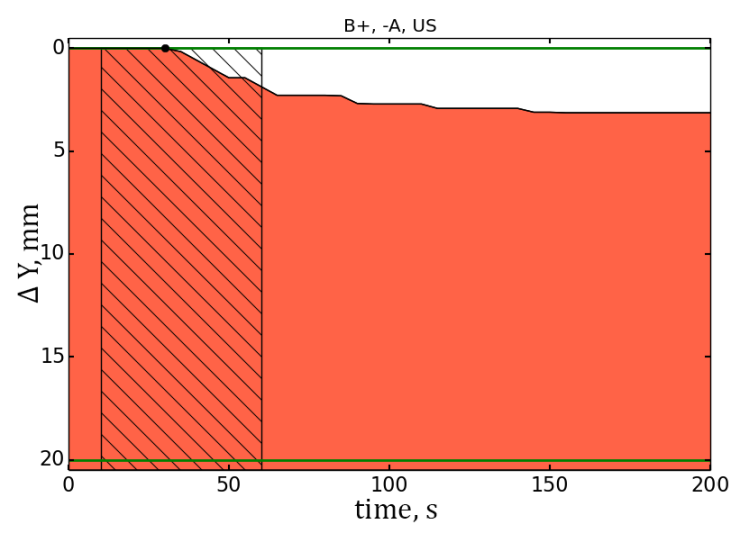

f

Fig. 6 Time dependence of the "supernatant - sediment" boundary displacement. (b), (c), (e), (f) correspond to the photographic images shown in Figs. 5 (b), (c), (e), (f) respectively. 1 - supernatant (plasma solution); 2 - sediment (erythrocyte mass), 3 - line corresponding to the meniscus of fluid in the cuvette - the level of the blood sedimentation start, 4 - line corresponding to the lower limit of the processing zone of experimental results W. The shaded area in (c) and (f) shows the time interval of ultrasonic irradiation of the sample. Note that the ultrasound was switched on 10 sec after the start of the video.

That is why small agglutinates are formed, which are suspended for a long time (several minutes). And only when small agglutinates combine into larger ones, a rather intensive process of agglutination and, consequently, their sedimentation starts. This interpretation is supported by Fig. 6f - here blood sedimentation takes place immediately, even during the process of ultrasound irradiation, so before it is turned off, since the aggregates (not agglutinates) created by ultrasound are larger than spontaneous (without ultrasound) agglutinates.

\subsection{The principle of photographic images processing in the absence of the "supernatant - sediment" interface}

The particular interest is the sedimentation of erythrocyte associates at a positive agglutination reaction in the presence of a standing ultrasonic wave (Fig. 5c). It is characteristic that in this case the agglutinates of large size are formed so extremely quickly that the photographic image of the suspension under study already at $10-20 \mathrm{sec}$ after the ultrasound is turned off has a discrete structure Fig. 7a and the boundary "supernatant - sediment" is completely absent - Fig. 5c, as well as Fig. 7a. Naturally, without such a boundary it is not possible to estimate the blood sedimentation rate in the traditional way. On the other hand, agglutinates of different sizes and shapes sediment in different ways and at different rates. Under these conditions, it was proposed to determine the sedimentation rate of agglutinates by the rate of change in the integral area $\mathrm{S}$ occupied by all agglutinates of the investigated zone $\mathrm{W}$ for the photographic image under analysis $(200 \times 650$ pixels $)$. It can be seen from Fig. 7 (1a-4a) that in the process of agglutinates sedimentation, the integral area $\mathrm{S}$ decreases in time, so that $\mathrm{S}$ is a function of time $\mathrm{t}$, and the derivative of this function $\Delta \mathrm{S} / \Delta \mathrm{t}$ can be taken as the rate of blood sedimentation.

The calculation of $\mathrm{S}(\mathrm{t})$ for each photo image of the video was carried out in accordance with the algorithm: 
- in the general photo of the cuvette with the blood solution under study, the zone $\mathrm{W}$ was highlighted (Fig. 7 (1a)) so that the probing light beam was located in its center; its position and size did not change when the subsequent photographs were under processing;

computer scanning of the zone $\mathrm{W}$ along the $\mathrm{X}$ and $\mathrm{Y}$ axes made it possible to determine the number of pixels, their coordinates in $\mathrm{W}$ zone, as well as the values of their brightness $\mathrm{B}(0<\mathrm{B}<255$ units of brightness); thus, the result of scanning the photo image $\mathrm{W}$ was a three-dimensional array of $\mathrm{X}, \mathrm{Y}, \mathrm{B}$;

- the average brightness of the pixels which corresponded to erythrocytes and their associates $B_{\text {er, }}$ as well as the same parameter for the "background" Bbg (space free from cells) were experimentally determined;

- then for each real photographic image $\mathrm{W}$ for a certain moment in time (for example, Fig. 7 (1a)), a corresponding artificial, equivalent image $\mathrm{W}_{\text {eq }}$ was constructed (for example, Fig. 7 (1b));

- $\quad$ for this purpose, an auxiliary image $\mathrm{W}_{\text {aux }}$ was preliminarily created with the dimensions identical to the processing area $\mathrm{W}_{\mathrm{aux}}=\mathrm{W}$; moreover, the brightness of each pixel of this auxiliary image $\mathrm{W}_{\text {aux }}$ was set equal to $\mathrm{B}_{\mathrm{bg}}$ for the green RGB component; this image $\mathrm{W}_{\text {aux }}$ will serve as a background for the equivalent photographs constructing for various frames;

- according to the results of $\mathrm{B}_{\mathrm{er}}$ and $\mathrm{B}_{\mathrm{bg}}$ measurements, a certain value of the threshold brightness $\mathrm{B}_{\mathrm{tb}}$ was set so that $\mathrm{B}_{\mathrm{er}}<\mathrm{B}_{\mathrm{tb}}<\mathrm{B}_{\mathrm{bg}}$;

- the number of pixels on the photographic image area $\mathrm{W}$ (in any photo frame), for which $\mathrm{B}_{\mathrm{er}}<\mathrm{B}_{\mathrm{tb}}$ was asumed as the number of pixels, which corresponded to erythrocyte aggregates or agglutinates $\mathrm{N}_{\mathrm{er}}$ (individual erythrocytes could not be visualized due to their relatively small size and insufficient resolution of the experimental setup); naturally, over time, from frame to

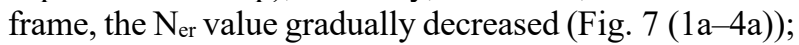

- then, for each real photo frame, the number of pixels $\mathrm{N}_{\mathrm{er}}$ counted was transferred on to the corresponding auxiliary background image $\mathrm{W}_{\mathrm{bg}}$; the transfer was carried out from bottom to top, and each such pixel was assigned a minimum brightness $\mathrm{B}_{\text {min }}$; as a result, a dark area was formed in the lower part of the equivalent image, which illustrated the sediment; then the upper area symbolized the supernatant (Fig. 7b);

- thus, each real photographic image with a discrete structure of chaotically located erythrocyte associates (for example, Fig. 7 (1a)) was assigned an equivalent image (for example, Fig. 7 (1b)), and the area of the "sediment" was equal to the area of all elements of the discrete structure, that is, the areas of all associates.

- As a result, the photographic image, on which there is no real boundary "sediment - supernatant" (for example, Fig. 7 (1a)), was transformed to an equivalent form for which such a boundary exists (for example, Fig. 7 (1b)). Further, the "equivalent" images for each photo frame (for example, Figs. $7(1 \mathrm{~b}-4 \mathrm{~b})$ ) were processed by analogy with the photo images of objects sedimentation with a real "supernatant-sediment" boundary (Section 4.3). The results of such processing are shown on Fig. 6c. By analogy with other Fig. 6, it is possible to determine the equivalent blood sedimentation rate $\mathrm{veq}$ for the case of a positive agglutination reaction when the object is exposed to an ultrasonic field.

\section{Discussion of the Results}

The order of magnitude of blood sedimentation rates for various experimental conditions is determined. In general, the results are summarized in Table 2 .

Table 2 - Sedimentation rate comparison under different experimental conditions.

\begin{tabular}{|c|c|c|c|c|c|c|}
\hline Object & $\begin{array}{l}\text { The properties } \\
\text { of the object }\end{array}$ & $\begin{array}{l}\text { Whole blood } \\
\text { content (\%) }\end{array}$ & $\begin{array}{l}\text { Sonication } \\
\text { time (sec) }\end{array}$ & $\begin{array}{l}\text { Average rate of object } \\
\text { sedimentation } \mathrm{v} / \mathrm{veq} \\
(\mathrm{mm} / \mathrm{min})\end{array}$ & $\begin{array}{l}\mathrm{v}_{1} \\
(\mathrm{~mm} / \mathrm{min})\end{array}$ & $\begin{array}{l}\text { V2 } \\
(\mathrm{mm} / \mathrm{min})\end{array}$ \\
\hline \multirow{6}{*}{$\begin{array}{l}\text { Blood } \\
\text { solution }\end{array}$} & \multirow{6}{*}{$\begin{array}{l}\text { Dilution by } \\
\text { saline (Fig. 3) }\end{array}$} & \multirow{2}{*}{0.25} & - & 0.09 & & \multirow{6}{*}{ - } \\
\hline & & & $30-90$ & 0.12 & & \\
\hline & & \multirow{2}{*}{$30-50$} & - & \multirow{2}{*}{0.03} & & \\
\hline & & & $30-90$ & & & \\
\hline & & \multirow{2}{*}{100} & - & 0.2 & & \\
\hline & & & $30-90$ & 0.14 & & \\
\hline \multirow{4}{*}{$\begin{array}{l}\text { Blood } \\
\text { solution } \\
+ \\
\text { tsoliklon }\end{array}$} & \multirow{2}{*}{$\begin{array}{l}\text { Negative } \\
\text { agglutination } \\
\text { reaction }\end{array}$} & \multirow{4}{*}{2} & - & 0.07 (Fig. 6e) & 0 & - \\
\hline & & & 50 & 0.8 (Fig. 6f) & 2.5 & 0.47 \\
\hline & \multirow{2}{*}{$\begin{array}{l}\text { Positive } \\
\text { agglutination } \\
\text { reaction }\end{array}$} & & - & 6 (Fig. 6b) & 2.3 & - \\
\hline & & & 50 & 14 (Fig.6c) & 7 & 9.22 \\
\hline
\end{tabular}


Note that in the presence of a supernatant - sediment interface, the blood sedimentation rate was determined as the rate of the displacement of this boundary $\mathrm{v}=\Delta \mathrm{Y} / \Delta \mathrm{t}$ using the results of photo processing (Fig. 3; Fig. 6 (b, e, $\mathrm{f})$. In this case, the value of $\Delta \mathrm{t}$ was taken as the time interval from the moment of the beginning of the boundary motion up to the moment of time when blood sedimentation process is almost complete, the sedimentation rate is close to zero. As noted in Section 4.3 and it can be seen from Fig. $6 \mathrm{f}$ and Fig. 6c, the sedimentation may start even before the ultrasound is turned off, if sufficiently large erythrocyte associates are formed under the action of the ultrasound wave and the condition for their levitation is violated.

If there is no supernatant-sediment interface (a positive agglutination reaction in the presence of ultrasound, Fig. 5c and Fig. 6c), in the Section 4.4 the concept of an equivalent blood sedimentation rate was $\mathrm{V}_{\mathrm{eq}}$ introduced. Table 2 shows the values of real blood sedimentation rate $\mathrm{v}$ but in the case of a positive reaction in the presence of ultrasound the value of $v_{\text {eq }}$ is given (Fig. 6c).

It should be noted that the sedimentation rates in Table 2 give a qualitative, comparative idea about the order of these values only. This is due to the fact that the values of the velocities depend on many experimental factors, as well as on the approach to make such estimations. In the last column of Table 2 the references to the figures show on what basis the blood sedimentation rates were calculated.

Table 2 shows the following.

- In the absence of monoclonal substances (tsoliklon) RBC associates sedimentation rate $\mathrm{v}$ rather weakly depends on the presence or absence of ultrasound and the duration of its action. Indeed, this rate is determined by the initial erythrocytes concentration of in the suspension to a greater extent. The minimum speed $\mathrm{v} \approx 0.03 \mathrm{~mm} / \mathrm{min}$ corresponds to a blood concentration in the suspension of $30-50(\%)$. A decrease in concentration, like an increase in it, increases the speed value; explained in Section 3.

- For a negative agglutination reaction, the role of ultrasound increases significantly. Thus, the sedimentation rate of RB associates increases by more than an order (from 0.07 to $0.8 \mathrm{~mm} / \mathrm{min}$ ) due to the action of an ultrasonic standing wave. It seems quite natural that the erythrocytes grouping in the nodes of the standing ultrasound wave increases the probability of their aggregation (not agglutination).

- $\quad$ For a positive agglutination reaction, the blood sedimentation rate in the presence of ultrasound is more than twice higher the same rate but without ultrasonic action. However, we note that from the point of view of the development of acousto-optical method for blood typing, it is more important to compare the rates of its sedimentation at positive and negative agglutination reactions.

- In the presence of ultrasound, the blood sedimentation rate for a positive agglutination reaction
$\mathrm{V}_{\text {eq }}+$ is significantly higher than the speed for a negative one $\mathrm{v}_{-}\left(\mathrm{V}_{\text {eq }}+\mathrm{v}_{-}\right) \approx 18$;

- To more precisely comparison of sedimentation process two more speed parameters $\mathrm{v}_{1}$ and $\mathrm{v}_{2}$ were calculated:

$\mathrm{V}_{1}$ - speed that was calculated on time range from start of sedimentation to the end of this process for positive agglutination reaction (Fig. 6c).

$\mathrm{V}_{2}$ - speed parameter for cases with US sonication. Time range: from US switching off till the end of sedimentation for positive agglutination reaction (Fig. 6c).

These parameters ( $\mathrm{v}_{1}$ and $\mathrm{v}_{2}$ ) are more suitable for comparison of sedimentation rates for cases which illustrated on Fig. 6. The difference between v1 and v2 for cases with US sonication shows that during sonication sedimentation goes faster for negative reaction, and slower for positive one than after US switching off. It is caused by RBC aggregation and agglutination: sonication increases process of RBC associates forming. After US switching off most of RBC aggregates are destructed and became a separate $\mathrm{RBC}$ or smaller aggregates unlike RBC agglutinates that still formed. This determines the sedimentation character after US switching off: for negative reaction it is slow and closer to the case for negative reaction without US sonication; for positive reaction it is rapid process caused by large RBC agglutinates sedimentation.

The latter circumstance determines a high level of the resolving power of the acousto-optical method for blood typing (Section 6) and, consequently, the reliability of its results.

\section{Resolution of the acousto-optical method to determine blood group type according to the $\mathrm{ABO}$ system}

Such a significant difference in the sedimentation rate of erythrocyte agglutinates (positive reaction) from the sedimentation rate of free erythrocytes and their aggregates (negative reaction) at the ultrasonic action on the blood sample under study makes it possible to obtain a high level of resolution $\mathrm{R}$ in blood group determination. It may be considered that this parameter is responsible for the reliability of the blood group typing. There are different ways to introduce this parameter, for example, $[18,23-25,28-31]$. At any rate $\mathrm{R}$ shows how strongly the physical quantity measured by the device in the case of a positive agglutination reaction differs from the same value, but with a negative one. Naturally, the reliability of blood group determination increases with the increase of R.

Some examples of the experimentally obtained resolution values of the acousto-optical method for blood group determination are given in Table 3 . It should be understood that the resolution $\mathrm{R}$ depends not only on the peculiarities of "blood solution-hemagglutinating serum (monoclonal substance)" interaction, but on the method of recording the result of the reaction also, as well 
Table 3 - Comparison of optical methods resolution power.

\begin{tabular}{|c|c|c|c|c|c|}
\hline № & $\begin{array}{l}\text { Optical method of } \\
\text { investigation }\end{array}$ & $\begin{array}{l}\text { Method of } \\
\text { resolution power } \\
\text { form }(\mathbf{R})\end{array}$ & Negative reaction & Positive reaction & $\begin{array}{l}\mathrm{R} \text {, literary } \\
\text { reference }\end{array}$ \\
\hline & 1 & 2 & 3 & 4 & 5 \\
\hline \multirow{3}{*}{ A } & \multirow{3}{*}{$\mathrm{RBC}$ sedimentation } & $\begin{array}{l}\text { Photometry: } \\
\text { Rmean }=\mathrm{B}_{+, \text {mean }} / \mathrm{B}_{- \text {,mean }}\end{array}$ & \multirow[b]{3}{*}{ a) } & \multirow[b]{3}{*}{ 5 } & $150[28]$ \\
\hline & & $\begin{array}{l}\text { Statistics: } \\
\mathrm{R}_{\mathrm{D}}=\mathrm{D}_{+} / \mathrm{D}_{-}\end{array}$ & & & \multirow{2}{*}{$\frac{328[28]}{2400[28]}$} \\
\hline & & $\begin{array}{l}\text { Discrete analysis } \mathrm{R}_{\Sigma} \\
=\mathrm{B}_{+, \Sigma} / \mathrm{B}_{-, \Sigma}\end{array}$ & & & \\
\hline B & Flow cytometry & $\begin{array}{l}\text { Area: } \\
\mathrm{R} \\
=\mathrm{S}_{+ \text {mean }} / \mathrm{S}_{- \text {,mean }}\end{array}$ & & & $\begin{array}{c}500-10000 \\
{[24]}\end{array}$ \\
\hline $\mathrm{C}$ & Digital microscopy & $\begin{array}{l}\text { Area: } \\
\mathrm{R} \\
=\mathrm{S}_{+, \text {mean }} / \mathrm{S}_{-, \text {mean }}\end{array}$ & & & $\begin{array}{c}1500-3000 \\
{[23]}\end{array}$ \\
\hline
\end{tabular}

as the method of processing the resulting photographic images.

Comments to the Table 3.

- The column 1 demonstrates the optical approach to register the presence or absence of the erythrocyte agglutination (the corresponding photo images are given in the columns 3 and 4). So, for example, the cell A-1 corresponds to the photographing of erythrocytes for a positive and negative reactions during blood sedimentation; the values measured were the magnitudes of photographic images brightness $\mathrm{B}_{+, \text {mean }}$ and $\mathrm{B}_{- \text {,mean }}$ averaged over the area $\mathrm{W}$ of the image - photometry. During the statistic processing of the same photographs, the distributions of pixels' brightness of photographs obtained were determined and compared: $\mathrm{D}_{+}$and $\mathrm{D}_{-}$are the values of the corresponding variances. The discrete analysis suggested:

1) either the calculation of the number of pixels with brightness above (or below) a given threshold of brightness value;

2) either the calculation of the total brightness values of all the pixels of the same photographic images, both for positive and negative agglutination reactions.

In Table 3, in particular, $\mathrm{B}_{+, \Sigma}$ and $\mathrm{B}_{-, \Sigma}$ are entered the values of the total brightness of the pixels of the photographic images for positive and negative reactions, respectively.

- $\quad$ Flow cytometry (line B) allows to obtain and to compare the photographic images of two types of suspensions (positive and negative reactions) when they flow through a glass capillary of a small internal size:
$\mathrm{S}_{+, \text {mean }}$ and $\mathrm{S}_{-, \text {mean }}$ are the mean values of the agglutinates areas or erythrocyte aggregates (and artifacts) in the corresponding photographs (the cells B-3 and B-4 of Table 3, respectively).

- Digital microscopy - photo registration of agglutinates (the cell B-3) and free erythrocytes and their aggregates (the cell $\mathrm{B}-4$ ). Here $\mathrm{S}_{+, \text {mean }}$ and $\mathrm{S}_{- \text {,mean }}$ are the mean values of the agglutinates areas and of erythrocytes (aggregates), respectively.

The Column 5 lists the corresponding values of the resolutions $\mathrm{R}$, as well as the corresponding literature references to the experimental works.

\section{Conclusion}

In accordance with the purpose of the work, an experimental study of the sedimentation of erythrocytes and their associates (aggregates or agglutinates) after ultrasonic irradiation was carried out in comparison with a similar experiment, but without acoustic action. The magnitudes of blood sedimentation rates were experimentally determined for the cases:

- blood solutions of various dilution levels in saline (with / without ultrasound);

- blood solutions containing naturally formed RBC aggregates, as well as erythrocyte aggregates formed by ultrasound standing wave for a negative agglutination reaction;

- erythrocyte agglutinates formed with / without the participation of an ultrasonic wave. 
It is shown that, in the absence of a standing ultrasonic wave, the blood sedimentation rate in the case of a positive agglutination reaction is almost two orders of magnitude higher than for a negative reaction. At the same time, ultrasonic action additionally more than doubles the blood sedimentation rate for a positive agglutination reaction. What is important for the development of an acousto-optical method to determine the blood group type: when the solution of blood sample under test is exposed by a standing ultrasound wave, the rate of blood sedimentation for a positive agglutination reaction is approximately 18 times higher than for a negative one.

Such a high difference in blood sedimentation rates at positive and negative agglutination reactions leads to a significant difference in the optical energetic characteristics of the samples under study. It is shown that the brightness of blood sample photo image or another measured parameter for a positive agglutination reaction may be some orders of magnitude higher than the same parameter of the sample for a negative reaction. This principle is the base of acousto-optical method for instrumental determination of the blood sample group.
The studies carried out are useful to understand the physical fundamentals of the acousto-optical method for the blood typing and the reasons for the high level of its resolution achieved. From a practical point of view, the experiments, which describe the sedimentation kinetic of $\mathrm{RBC}$ and their associates are of most interest, especially for the cases of positive and negative agglutination reactions under the ultrasonic action. These experiments make it possible to estimate the required duration of ultrasonic action upon the blood tested, as well as the time duration of its incubation - the time interval from the moment of the end of the ultrasound irradiation to the moment of reading the result. This is all the more important if the proposed acousto-optical method will be used as the basis to create the automatic device for blood typing.

\section{Disclosures}

All authors declare that there is no conflict of interests in this paper.

\section{References}

1. W. L. Nyborg, Ultrasound: Its application in medicine and biology, Elsevier, New York (1978).

2. G. Whitworth, M. A. Grundy, and W. T. Coakley, "Transport and harvesting of suspended particles using ultrasound," Ultrasonics 29(6), 439-444 (1991).

3. C. J. Schram, "Manipulation of particles in an acoustic field," Adv. Sonochem, 2, 293 (1991).

4. W. T. Coakley, G. Whitworth, M. A. Grundy, R. K. Gould, and R. Allman, "Ultrasonic manipulation of particles and cells," Bioseparations 4(2), 73-83 (1994).

5. W. T. Coakley, D. W. Bardsley, M. A. Grundy, F. Zamani, and D. J. Clarke, "Cell manipulation in ultrasonic standing wave field," Journal of Chemical Technology \& Biotechnology 44(1), 43-62 (1989).

6. M. S. Limaye, J. J. Hawkes, and W. T. Coakley, "Ultrasonic standing wave removal of microorganisms from suspension in batch systems," Journal of microbiological methods 27(2-3), 211-220 (1996).

7. W. T. Coakley, D. W. Bardsley, "Enhanced sedimentation of mammalian cells following acoustic aggregation," Biotechnology and bioengineering 34(4), 559-562 (1989).

8. O. Doblhoff-Dier, T. Gaida, H. Katinger, W. Burger, M. Groschl, and E. Benes, "A novel ultrasonic resonance field device for the retention of animal cells," Biotechnology Progress 10(4), 428-432 (1994).

9. J. J. Hawkes, W. Coakley, “A continuous flow ultrasonic cell filtering method," Enzyme and Microbial Technology 19(1), 57-62 (1996).

10. J. J. Hawkes, M. S. Limaye, and W. T. Coakley, "Filtration of bacteria and yeast by ultrasound-enhenced sedimentation," Journal of Applied Microbiology 82(1), 39-47 (1997).

11. F. Trampler, S. A. Sonderhoff, P. W. S. Pui, D. G. Kilburn, and J. M. Piret, "Acoustic cell filter for high density perfusion of culture of hybridoma cells," Bio/technology 12(3), 281-284 (1994).

12. C. A. Miles, M. J. Morley, W. R. Hudson, and B. M. Mackey, "Principles of separating microorganisms from suspensions using ultrasound," Journal of Applied Bacteriology 78(1), 47-54 (1995).

13. A. N. Alipov, V. Z. Vaninskiy, L. B. Denisov, S. I. Donskov, V. A. Dubrovskiy, E. N. Zav’yalov, and N. N. Knyazkov, "Method for determining the agglutination reaction," Inventor's Certificate of the invention №1683760, 04.06.1987. [in Russian].

14. M. A. Grundy, W. E. Bolek, W. T. Coakley, and E. Benes, "Rapid agglutination testing in an ultrasonic standing wave field," Journal of Immunological Methods 165(1), 47-57 (1993).

15. M. A. Grundy, K. Moore, and W. T. Coakley, "Increased sensitivity of diagnostic latex agglutination tests in an ultrasonic standing wave field," Journal of Immunological Methods 176(2), 169-177 (1994).

16. E. E. Pohl, E. H. Rosenfeld, P. Pohl, and R. Millner, "Effects of ultrasound on agglutination and aggregation of human erythrocytes in vitro," Ultrasound in Medicine \& Biology 21(5), 711-719 (1995).

17. E. T. Nerys, W. T. Coakley, "Measurement of antigen concentration by an ultrasound-enhanced latex immunoagglutination assay," Ultrasound in Medicine \& Biology 22(9), 1277-1284 (1996). 
18. V. A. Doubrovski, K. N. Dvoretski, "Ultrasonic wave action upon the red blood cell agglutination in vitro," Ultrasound in Medicine \& Biology 26(4), 655-659 (2000).

19. V. A. Doubrovski, K. N. Dvoretski, and A. E. Balaev, "Mechanism of erythrocyte aggregation enhancement by ultrasonic field," Acoustic Journal 50(2), 146-153 (2004).

20. K. N. Dvoretski, Increasing the resolution of the photometric method for recording agglutination of human erythrocytes in vitro, PhD Thesis, Saratov State University, Saratov (2004).

21. V. A. Doubrovski, A. A. Dolmashkin, "Human blood group typing based on digital photographs of RBC agglutination process," Journal of Optics and Spectroscopy 109(2), 236-267 (2010).

22. V. A. Doubrovski, A. A. Dolmashkin, "Blood group typing based on digital imaging of sedimentation of erythrocytes and their agglutinates," Biomedical Engineering 46(2), 65-70 (2012).

23. Yu. A. Ganilova, V. A. Doubrovski, and I. V. Zabenkov, "The resolving power of the flowing method to register the process of human erythrocytes agglutination in vitro on the base of correlation analysis of microphotographs," Proceedings of SPIE 7999, 799903 (2011).

24. V. A. Doubrovski, Yu. A. Ganilova, and I. V. Zabenkov, “Application of a spectrally filtered probing light beam and $\mathrm{rgb}$ decomposition of microphotographs for flow registration of ultrasonically enhanced agglutination of erythrocytes," Optics and Spectroscopy 115(2), 218-227 (2013).

25. V. A. Doubrovski, I. V. Zabenkov, and S. O. Torbin, "Blood group typing in the ab0 system using digital microscopy," Biomedical Engineering 47(3), 126-129 (2013).

26. Yu. A. Ganilova, V. A. Doubrovski, and I. V. Zabenkov, "R and G color components competition of RGB image decomposition as a criterion to register RBC agglutinates for blood group typing," Journal of Biomedical Optics 19(3), 036012 (2014).

27. V. A. Doubrovski, M. F. Medvedeva, "The acousto-optical method for blood typing (part 1). photometric and statistical methods for image processing," Biomedical Engineering 50(1), 10-14 (2016).

28. V. A. Doubrovski, M. F. Medvedeva, "The acousto-optical method for blood typing based on discrete processing of photographic images," Biomedical Engineering 50(2), 78-83 (2016).

29. V. A. Doubrovski, M. F. Medvedeva, S. O. Torbin, and K. N. Dvoretskiy, "Visualization of RBC grouping by ultrasonic standing wave in vitro," in Problems of Optics and Biophotonics, 20-28 (2014) [in Russian].

30. V. A. Doubrovski, C. N. Dvoretski, S. V. Markov, E. P. Karpocheva, and V. V. Tuchin, "Optical Digital Registration of Erythrocyte Sedimentation and Its Modeling in the Form of the Collective Process," Optics and Spectroscopy 126(5), 595-606 (2019).

31. R. A. Steven, A Simplified Visible/Near-Infrared Spectrophotometric Approach to Blood Typing for Automated Transfusion Safety, North Carolina State University, Raleigh (2005).

32. J. B. Lambert, A miniaturized device for blood typing using a simplified spectrophotometric approach, North Carolina State University, Raleigh (2006). 